\title{
Open conformation of hERG channel turrets revealed by a specific scorpion toxin BmKKx2
}

\author{
You-Tian $\mathrm{Hu}^{1}$, Jun $\mathrm{Hu}^{1}$, Tian $\mathrm{Li}^{1}$, Jing-Jing Wei ${ }^{1}$, Jing Feng ${ }^{1}$, Yi-Mei Du², Zhi-Jian Cao ${ }^{1}$, Wen-Xin $\mathrm{Li}^{{ }^{*}}$ \\ and Ying-Liang $\mathrm{Wu}^{1 *}$
}

\begin{abstract}
Background: The human ether-a-go-go-related gene potassium channel (hERG) has an unusual long turret, whose role in recognizing scorpion toxins remains controversial. Here, BmKKX2, the first specific blocker of hERG channel derived from scorpion Mesobuthus martensii, was identified and the turret role of hERG channel was re-investigated using BmKKX2 as a molecular probe.

Results: BmKKx2 was found to block hERG channel with an $\mathrm{I}_{50}$ of $6.7 \pm 1.7 \mathrm{nM}$ and share similar functional surface with the known hERG channel inhibitor BeKm-1. The alanine-scanning mutagenesis data indicate that different residue substitutions on hERG channel by alanine decreased the affinities of toxin BmKK×2 by about 10-fold compared with that of wild-type hERG channel, which reveals that channel turrets play a secondary role in toxin binding. Different from channel turret, the pore region of $\mathrm{hERG}$ channel was found to exert the conserved and essential function for toxin binding because the mutant hERG-S631A channel remarkably decreased toxin BmKKx2 affinity by about 104-fold.

Conclusions: Our results not only revealed that channel turrets of hERG channel formed an open conformation in scorpion toxin binding, but also enriched the diversity of structure-function relationships among the different potassium channel turrets.
\end{abstract}

Keywords: Scorpion toxin, BmKKx2, hERG channel, Turret, Pore region, Molecular mechanism

\section{Background}

Potassium channels mediate $\mathrm{K}^{+}$efflux and play various pharmacological functions [1]. Structurally, the potassium channels present various topologies, such as inwardly rectifying two-transmembrane potassium channels (Kir), calcium-activated potassium channels with six or seven transmembrane segments ( $\mathrm{KCa})$ and voltage-gated potassium channels with six transmembrane segments (Kv) [2]. Due to the channel structure complexity and technique challenge, there are a few crystal structures of eukaryotic potassium channels, such as Kv1.2, Kir2.2 and Kir3.2 [3-5]. Comparison of these crystal structures reveals essential conformation differences in the extracellular pore entryway that includes turret and filter regions. In recent years, other structural features of channel turrets were also revealed by the specific scorpion toxins as the molecular probes. For example, the 10-residue turrets of

\footnotetext{
* Correspondence: liwxlab@whu.edu.cn; ylwu@whu.edu.cn

'State Key Laboratory of Virology, College of Life Sciences, Wuhan University, Wuhan 430072, China

Full list of author information is available at the end of the article
}

Kv1.2 channel is induced to form an open state conformation and not affect toxin binding activity when it is bound by scorpion toxin maurotoxin [6]. Different from the open state conformation of Kv1.2 channel turret, the 17-residue turrets of small conductance $\mathrm{KCa} 3$ (SKCa3) channel form a "peptide screener" compact conformation which selectively controls scorpion toxin binding [7]. More interestingly, the 19-residue turret of large conductance $\mathrm{KCa}(\mathrm{BKCa})$ channel switches from an open state conformation for scorpion toxin charybdotoxin (ChTX) binding to a compact "helmet" conformation for toxin ChTX insensitivity when the channel turret interacts with its auxiliary $\beta 4$ subunits [8]. Besides above progress of structure and function relationships of different potassium channel turrets, characterization of more potassium channel extracellular pore entryways remains a challenge nowadays due to the difficulties in determining the crystal structures of additional potassium channels.

Among all the superfamily members of potassium channels, hERG (human ether-a-go-go-related gene) potassium channel has an unusual longer turret containing 
40 amino acid residues [9]. Using the scorpion toxin BeKm-1 as a molecular probe, different conformational states in the turret of hERG channel have been reported. Through the computational simulation technique, our group ever predicted that hERG channel vestibule presented an open conformation mainly in a decentralized 'petunia' shape and turrets were far from the bound toxin BeKm-1 [10], while another model without a 'petunia' shape was also proposed by other group, in which channel turrets directly interacted with the bound BeKm-1 [11]. Thus, these controversial structural-functional features still need to be investigated using scorpion toxins as a molecular probe. In this work, we revisit the conformational state of hERG channel turrets by using a specific scorpion toxin BmKKx2, a novel toxin from Scorpion Mesobuthus martensii with similar structural and functional features as BeKm-1 [12]. Through alanine-scanning mutagenesis and pharmacological experiments, hERG channel pore region instead of turrets was mainly responsible for toxin BmKKx2 binding, supporting our previous inference that the hERG channel turret adopts an open conformation. Taken together, these findings provide new insights into the role of channel turret in toxin recognition and might help to better understand the structure-function relationship of hERG channel.

\section{Results}

\section{Expression and purification of BmKKx2}

As shown in Figure 1A, there are only two differential residues between BmKKx2 and BeKm-1, which suggested that BmKKx2 might also act as a hERG channel blocker [12,13]. To evaluate the pharmacological feature of BmKKx2, we obtained recombinant $B m K K x 2$ as described previously $[7,10]$.

The GST-BmKKx2, a fusion protein of $30 \mathrm{kDa}$ size, was purified and split into two products, GST of $26 \mathrm{kDa}$ and $\mathrm{BmKKx} 2$ of $4.1 \mathrm{kDa}$ (Figure 1B). The mixture was further separated by HPLC, and two product peaks were obtained (Figure $1 \mathrm{C}$ ). The component eluting at about $18 \mathrm{~min}$ corresponding to $\mathrm{BmKKx} 2$ was collected manually and lyophilized. By the matrix-assisted-laser-desorption/ionization time-of-flight mass spectrometry (MALDI-TOF-MS), the molecular weight of recombinant BmKKx2 was 3999.6 (Figure 1D). Considered the loss of $6 \mathrm{Da}$ by three pairs of the conserved disulfide bridges, the determined molecular weight was in good agreement with the theoretical molecular weight of $4005.6 \mathrm{Da}$ calculated by the ExPASy Protemics Server (http://us.expasy.org/tools/protparam.html).

\section{BmKKx2 appears to be a selective hERG channel blocker} The recognition process between toxin peptides and potassium channels remains interesting and complicated. A slight change of toxin peptides, even a single residue modified, may induce a distinct recognizing pattern [14-16]. Considering BmKKx2 as a new member of $\gamma-\mathrm{K}^{+}$-channel toxin subfamily, the blocking activity was evaluated on HEK293 cells that transiently expressed hERG channel. As shown in Figure 2A, BmKKx2 could effectively block hERG channel with an $\mathrm{IC}_{50}$ of $6.7 \pm 1.7 \mathrm{nM}$, which was comparable to that of BeKm-1, an effective blocker of hERG channel [12]. Furthermore, we also investigated its pharmacological effects on other potassium channels, including Kv1.1, Kv1.2, Kv1.3, Kv4.2 and SKCa2. The results revealed that $\mathrm{BmKKx} 2$ had less effect on these potassium channels even at a concentration of $1 \mu \mathrm{M}$ (Figure 2B-F). These data revealed BmKKx2 to be the first hERG channel selective inhibitor derived from scorpion Mesobuthus martensii, and making BmKKx2 a potential molecular probe of hERG channel.

Besides the selectivity of toxin BmKKx2, its functional surface was also investigated. Six candidate residues (Arg1, Tyr11, Lys18, Arg20, Lys23 and Leu32) were selected and respectively mutated to alanine according to the functional map of molecular surface of BeKm-1 [17], Compared with the wild-type BmKKx2, all mutants showed less significant changes in the secondary structure through CD spectra, indicating these mutants adopted the same overall structural topology with BmKKx2 (see Additional file 1: Figure S1). Next, the blocking activities of these mutant peptides were tested on hERG channel (Figure 3) and all these results were listed in Table 1. Compared with wild-type BmKKx2, BmKKx2-K18A and BmKKx2-R20A exhibited dramatic drop of affinity on hERG channels for 56- and 61-fold, respectively (Figure 3C,D and H). Furthermore, Tyr11 and Lys23 of BmKKx2 were found moderately important for binding (Figure 3B,E,G and H), while Arg1 and Leu32 exhibited less effect on toxin blocking activities (Figure 3A, $\mathrm{F}$ and G). All these data revealed that BmKKx2 mainly used Tyr11, Lys18, Arg20 and Lys23 as the functional residues to recognize hERG channel, which was similar to the binding surface of toxin BeKm-1.

\section{Less effect of channel turret on BmKKx2 binding}

With the help of specific scorpion toxin BmKKx2 as a molecular probe, the extracellular pore entryway role of hERG channel was revisited in this work. Different from the classic potassium channels, hERG channel has an unusual longer turret containing 40 amino acid residues (Figure 4), whose role remains confused in the toxinchannel interaction [10,11]. The middle part of turret (W585 Y597) was found to form a helix conformation in the hydrophobic environment but predominantly present a random coil structure in aqueous solution [18]. This possible helix divided the long turret into three regions: S5-helix linker region $(\mathrm{S} 5 \mathrm{H})$, helix region, helix-pore helix linker region (HP). Here, the roles of these three regions were respectively investigated during the toxin BmKKx2-hERG channel interaction. 


\section{A}

BmKKx2: RPTDIKCSASYQCFPVCKSRFGKTNGRCVNGLCDCF

BeKm-1: RPTDIKCSESYQCFPVCKSRFGKTNGRCVNGFCDCF
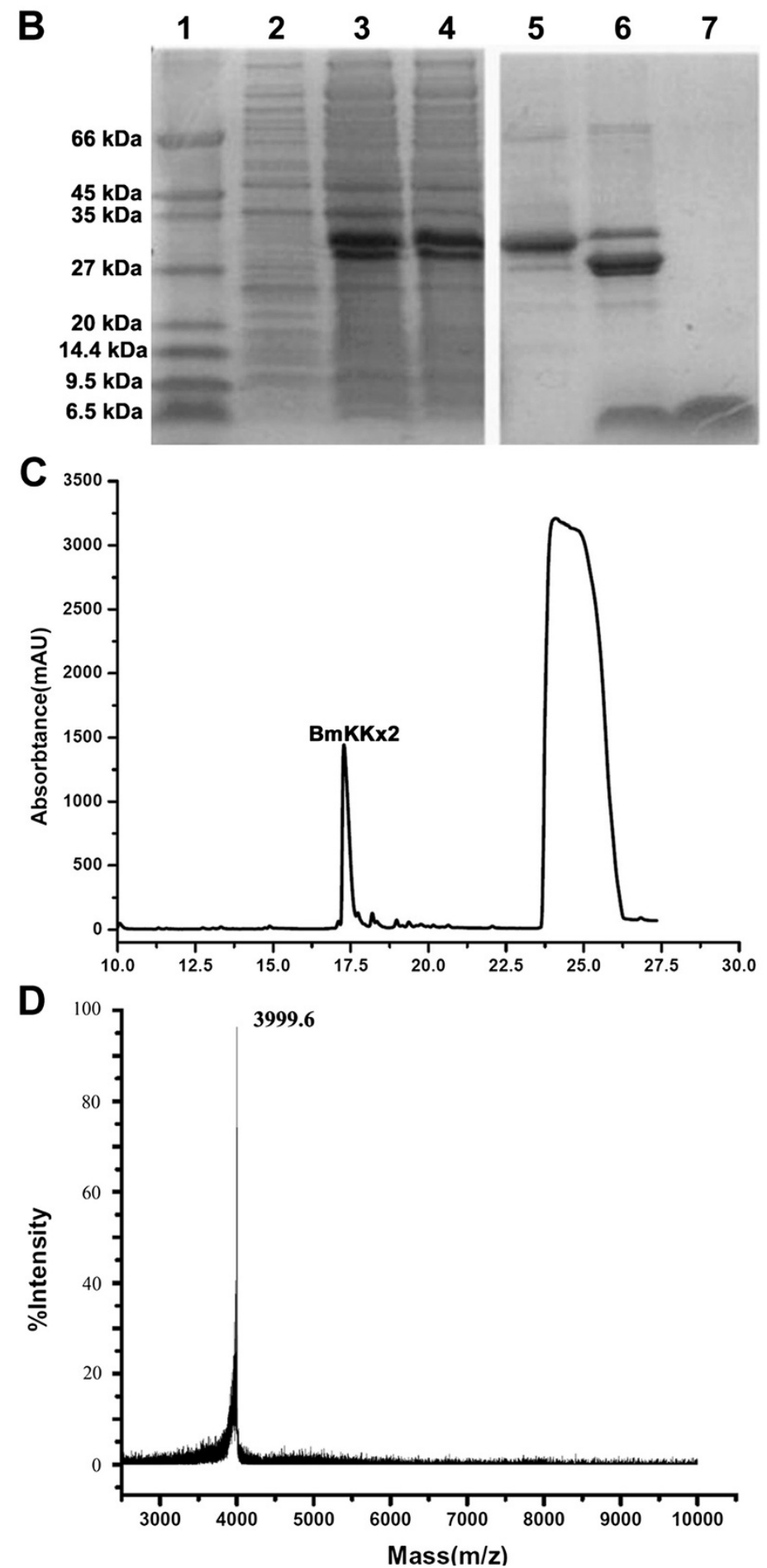

Figure 1 (See legend on next page.) 
(See figure on previous page.)

Figure 1 Purification and characterization of toxin BmKKx2. (A) Amino acid sequence alignment for BmKKx2 and BeKm-1. The differential residues were marked with asterisks. (B) Tricine-SDS-PAGE analysis of purified toxin BmKKx2. Lane 1, molecular mass markers; lane 2, non-induced cell-free extract of E. coli carrying pGEX-6p-1-BmKKx2; Lane 3, total cell-free extract of E. coli carrying pGEX-6p-1-BmKKx2 induced with IPTG for $4 \mathrm{~h}$; lane 4, the purified GST-BmKKx2 protein after affinity chromatography; lane 5, the purified GST fusion protein after desalting and concentration; lane 6, the cleaved fusion protein via enterokinase; lane 7, the purified toxin BmKKx2 through reversed phase HPLC. (C) HPLC profile of the GST-BmKKx2 fusion protein cleaved through enterokinase. (D) Mass spectrum of BmKKx2 peptide measured on MALDI-TOF-MS.

Firstly, the involvement of $\mathrm{S} 5 \mathrm{H}$ region (I571 G584) was identified using the alanine-scanning strategy. Since the N-terminal of $\mathrm{S} 5 \mathrm{H}$ region, near the channel S5 transmembrane helix (outer helix in Figure 4), is far from the central pore and likely plays an unimportant role in toxin binding, four residues (His578, Asp580, Arg582 and Ile583) in the C-terminal of $\mathrm{S} 5 \mathrm{H}$ region were selected and replaced by alanine, individually. It was found that the mutant channel hERG-H578A did not express and hERG-D580A mediated too small currents, making them inappropriate for evaluating their sensitivity towards BmKKx2. For the mutant channels hERG-R582A and hERG-I583A, about 70\% of the currents were inhibited by $100 \mathrm{nM}$ BmKKx2 (Figure 5A,B). As shown in Table 2, the dose-dependent inhibition of mutant channels by BmKKx2 indicated that the $\mathrm{IC}_{50}$ values of toxin $\mathrm{BmKKx} 2$ on hERGR582A and hERG-I583A channels were 76.5 and $87.5 \mathrm{nM}$, respectively (Figure 6A). In comparison with the wild-type hERG channel, two mutant hERG-R582A and hERGI583A channels respectively decreased the BmKKx2 sensitivity by 11.4- and 13.2-fold. These data showed that $\mathrm{S} 5 \mathrm{H}$ region had less effect on the toxin $\mathrm{BmKKx} 2$ binding, which was in line with the investigation by the homologous scorpion toxin BeKm-1 [17].

Secondly, we continued to investigate the effect of the controversial helix region in channel turrets on toxin BeKm-1 binding $[10,11]$. Except no expression of hERGW585A and lower expression level of hERG-L589A, other eight mutant channels (hERG-L586A, hERG-H58 7A, hERG-N588A, hERG-D591A, hERG-Q592A, hERGK595A, hERG-P596A and hERG-Y597A) exhibited the similar properties to that of wild-type hERG channels, and their effects on toxin BmKKx2 binding were determined (Figure $5 \mathrm{C}-\mathrm{J})$. The corresponding $\mathrm{IC}_{50}$ values of toxin BmKKx2 were 24.3, 59.7, 14.5, 19.6, 89.5, 65.2, 60.8 and 118.2 $\mathrm{nM}$ for hERG-L586A, hERG-H587A, hERG-N5 88A, hERG-D591A, hERG-Q592A, hERG-K595A, hERGP596A and hERG-Y597A, respectively (Figure 6A-C and Table 2). Overall, there were drops of no more than 20fold toxin sensitivity towards eight mutant hERG channels, which indicated that the helix region in channel turrets did not play an important role in scorpion toxin recognition.

Finally, the influence of the HP linker in channel turret in BmKKx2 recognition was also studied. Six mutant channels were constructed, and mutant hERG-D609A channel could not be investigated due to the negligibly small currents. Other five mutant channels (hERG-N598A, hERG-S600A, hERG-K608A, hERG-K610A and hERGT613A) were able to mediate potassium currents with comparable amplitude with wild-type hERG channel, and also were effectively blocked by $100 \mathrm{nM}$ toxin BmKKx2 (Figure $5 \mathrm{~K}-\mathrm{O}$ ). The $\mathrm{IC}_{50}$ values of toxin $\mathrm{BmKKx} 2$ for hERG-N598A, hERG-S600A, hERG-K608A, hERG-K610A and hERG-T613A were 15.2, 12.1, 116.1, 6.7 and 42.6 $\mathrm{nM}$, respectively (Figure $6 \mathrm{C}$ and D). Similar to the previous two $\mathrm{S} 5 \mathrm{H}$ and helix regions of the channel turret (Table 2), these experimental data of HP linker residues indicated that it also played a secondary role in the toxin BmKKx2 binding.

\section{Critical role of channel pore region in $\mathrm{BmKK} \times 2$ binding}

The interaction between animal toxin blockers and potassium channels indicated that the channel pore region played a critical role in toxin recognition $[19,20]$. In this work, two residues Asn629 and Ser631 near the channel selectivity filter were selected and substituted by alanine residue, respectively. Since the lower expression of the mutant channel hERG-N629A, only mutant hERG-S631A channel was investigated for toxin BmKKx2 recognition. As shown in Figure 5P, mutant hERG-S631A channel currents could be inhibited by $100 \mathrm{nM}$ toxin $\mathrm{BmKKx} 2$, and its $\mathrm{IC}_{50}$ of toxin $\mathrm{BmKKx} 2$ was $698 \pm 64.4 \mathrm{nM}$, making hERG-S631A approximately 104-fold less sensitive to BmKKx2 than wild-type hERG channel (Figure 6D). The remarkable effect of channel Ser631 on toxin binding confirmed that the channel pore region was greatly involved in toxin binding.

\section{Discussion}

With about 100 members, the potassium channels present the diverse structure-function relationships. The extracellular pore entryway including turrets and filter regions is the critical domain responsible for the inactivation, animal toxin recognition and interactions between channel subunits. Due to the difficulties in solving the crystal structures of potassium channels, the scorpion toxin blockers are useful molecular probes to characterize the structure-function relationships of the different channel extracellular pore entryways [6-8,20]. 


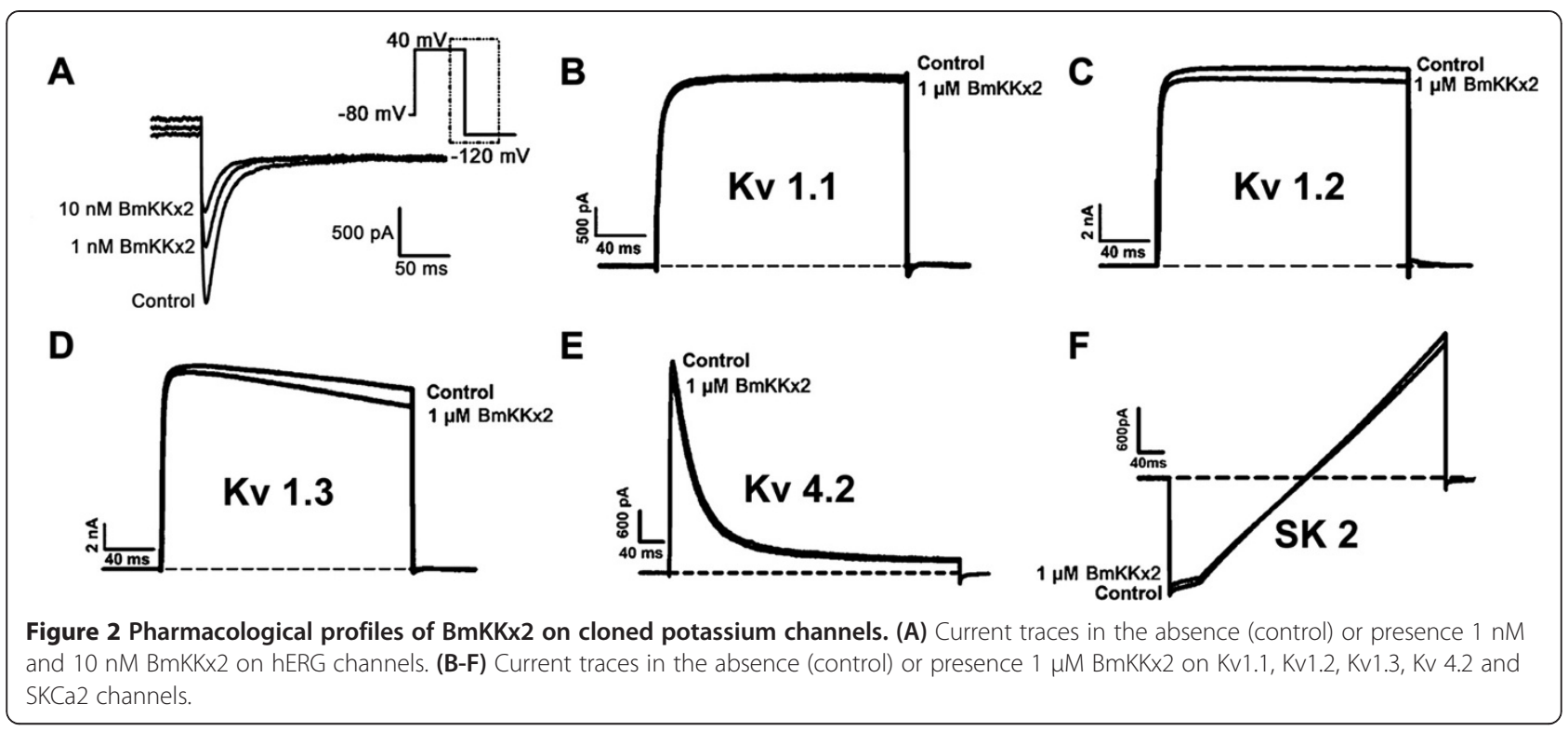

In this work, we revisited the hERG channel turret with an unusual longer turret containing 40 amino acid residues (Figure 4), whose role was ever intensively investigated in the scorpion toxin binding $[10,11,17]$. However, there were two different inferences on the orientation of hERG channel turrets: one is the helix domain of hERG channel turrets responsible for scorpion toxin binding through the cysteine-scanning mutagenesis and computational modeling [11], the other is the turret helix domain far from the bound scorpion toxin through the computational modeling [10]. Unexpectedly, the cysteine substitutions of hERG channel turrets at different positions were found to have high-impact, intermediate-impact or low-impact on channel functions and affect scorpion

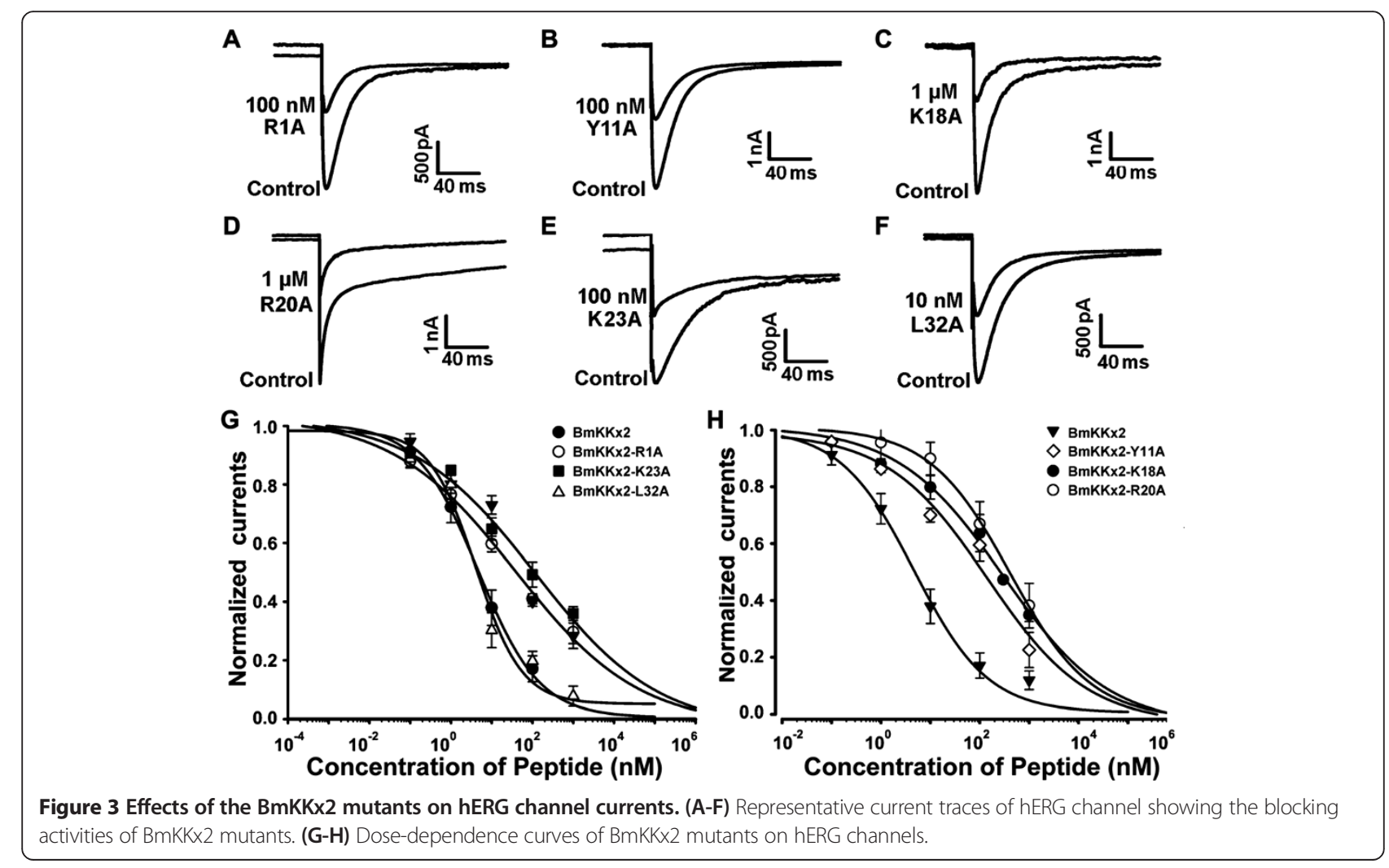


Table 1 The binding affinities of wild-type BmKKx2 and its mutants towards hERG channel

\begin{tabular}{cccc}
\hline BmKKx2 mutant & $\mathbf{I C 5 0}^{\boldsymbol{a}}$ & $\boldsymbol{n}^{\boldsymbol{b}}$ & $\mathbf{I C}_{\mathbf{5 0 ( \mathbf { m u t } )}} / \mathbf{I} \mathbf{C}_{\mathbf{5 0 ( w \mathbf { t } )}}$ \\
\hline Wild type & $\boldsymbol{n} \boldsymbol{M}$ & & \\
R1A & $26.7 \pm 1.7$ & 6 & 1.0 \\
Y11A & $238.3 \pm 11.2$ & 4 & 8.1 \\
K18A & $131.0 \pm 35.2$ & 4 & 27.9 \\
R20A & $376.6 \pm 62.0$ & 6 & 80.1 \\
F21A & $409.6 \pm 56.9$ & 5 & 87.1 \\
K23A & $863.4 \pm 73.4$ & 5 & 183.7 \\
R27A & $123.2 \pm 56.3$ & 4 & 26.2 \\
F32A & $262.7 \pm 25.9$ & 4 & 13.3 \\
\hline
\end{tabular}

${ }^{a} \mathrm{I}_{50}$ value(half-maximum inhibition concentration) represents as Mean \pm Standard Error. ${ }^{b}$ number of independent experiments.

toxin binding due to the potential formation of the disulfide bonds [11,22]. In order to avoid the effect of disulfide bonds on scorpion toxin binding, the alaninescanning mutagenesis was used to re-investigate the role of hERG channel turret, especially the controversial turret helix domain, in the scorpion toxin binding.

\section{Conserved functional surface of new toxin BmKKx2}

In order to explore the functional role of hERG channel turrets in scorpion toxin recognition, a homologous scorpion toxin BmKKx2 specific for hERG channel was identified with an $\mathrm{IC}_{50}$ of $6.7 \pm 1.7 \mathrm{nM}$ (Figure 2). Furthermore, the structure-function relationship of toxin $\mathrm{BmKKx} 2$ indicated that it shared the same critical residues (Tyr11, Lys18, Arg20 and Lys23) as those of nearly identical scorpion toxin BeKm-1 (Figures 3 and 7A-B, and Table 1) [12]. These results demonstrated that toxin $B m K K x 2$ was also a useful molecule probe to explore the functional role of hERG channel turrets in toxin recognition.

\section{Open conformation of hERG channel turrets in toxin BmKKx2 recognition}

As the role of channel turrets in scorpion toxin binding, the controversial issue was whether the helix domain interacted with the bound toxin (Figure 4) [10,11] although this helix domain actually did not exist due to locating above the extracellular pore entryway in the aqueous solution instead of the hydrophobic environment [9]. In previous cysteine-scanning experiments [11], total 29 mutant channels were investigated while

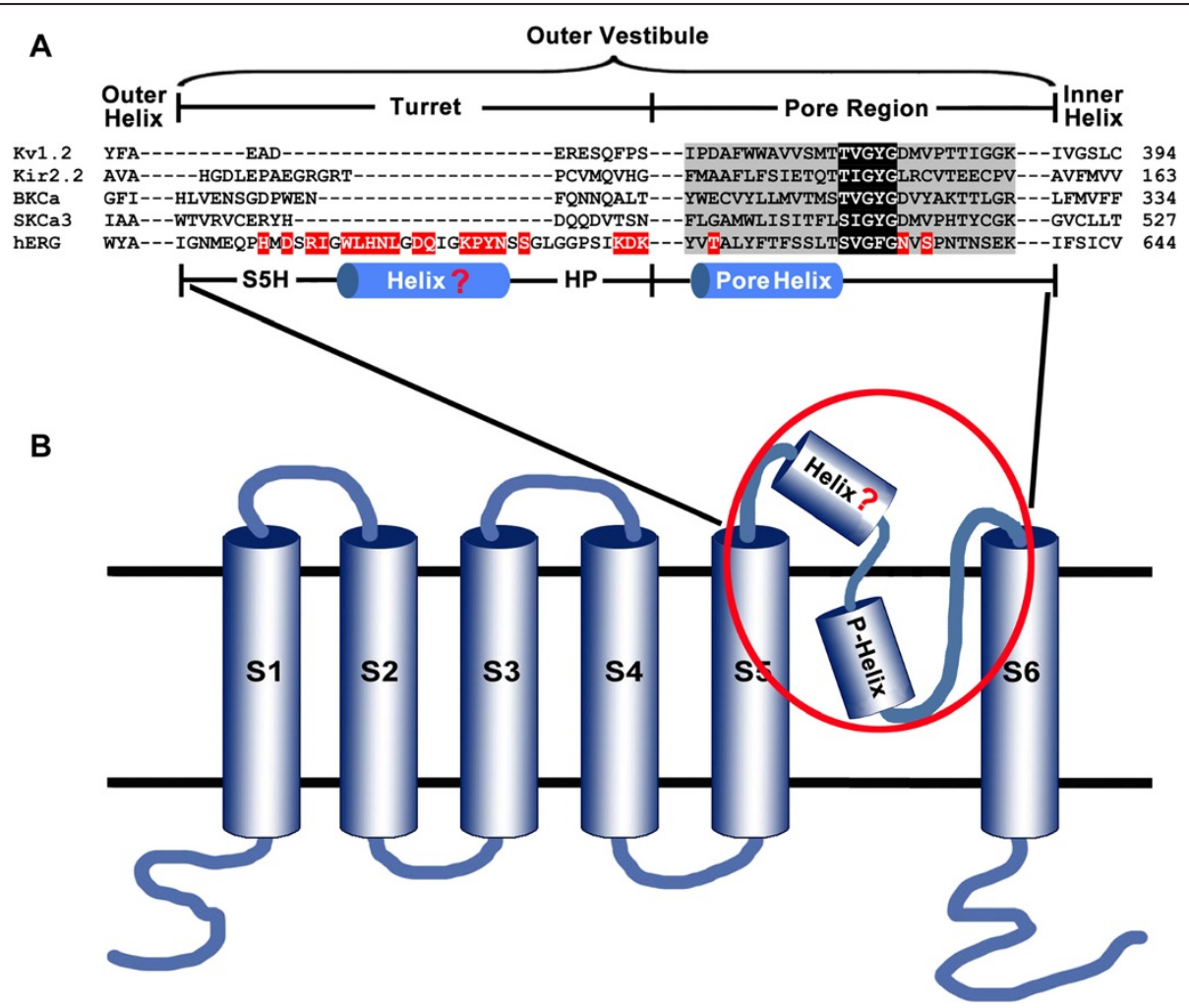

Figure 4 Alignment of the outer vestibules of hERG channel and other potassium channels. (A) Primary sequence alignment for hERG channel and other potassium channels. Black shading shows the selectively filter, whereas gray background indicates pore region of potassium channels, respectively. Red letters highlight the residues selected for alanine-scanning mutagenesis. These residues are H578, D580, R582, I583, W585, L586, H587, N588, L589, D591, Q592, K595, P596, Y597, N598, S600, K608, D609, K610, T613, N629 and S631. (B) Diagram of hERG channel subunit containing six transmembrane domains and possible helix structure in channel turret. 


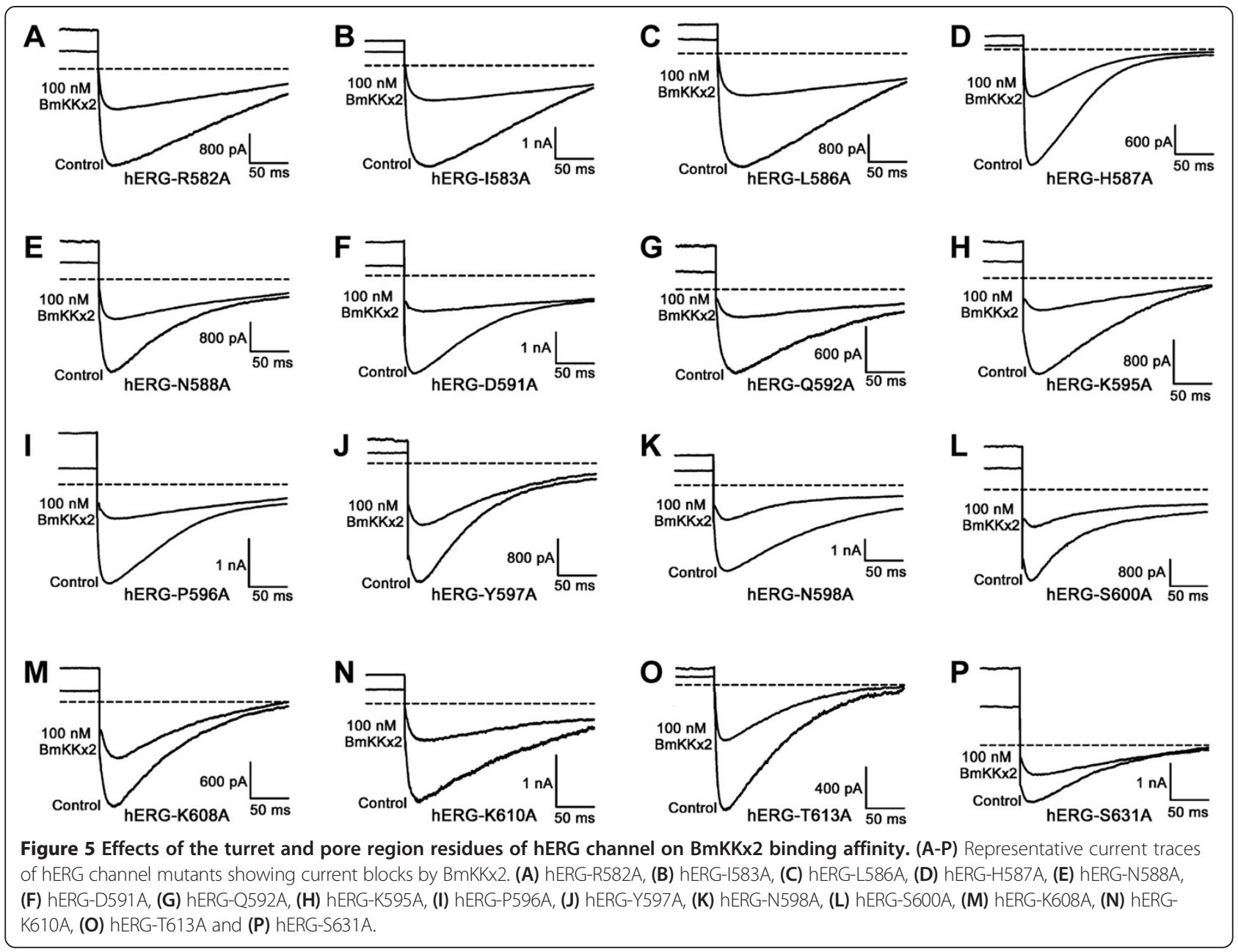

their cysteine substitutions had intermediate-impact or low-impact on the channel function [22]. It was found that the substitution of Gln592 by the cysteine in the helix domain significantly decreased the affinity of toxin BeKm- 1 by about 54 -fold, and the remaining 28 turret residue substitutions including three $\mathrm{S} 5 \mathrm{H}$, helix and $\mathrm{HP}$ regions (Figure 4) decreased toxin BeKm-1 affinities by less than 10-fold when compared with the that of wild-type hERG channel. These results supported the inference that helix domain of channel turrets would interact with toxin BeKm-1. In our work, 15 channel turret residues were successfully assessed through the alanine-scanning mutagenesis (Figures 5 and 6, and Table 2). Different from the important effect of channel turret Gln592 on toxin BeKm1 binding [11], there was only about 13-fold drop in toxin BmKKx2 binding affinity when Gln592 residue was replaced by alanine (Figures 5G, 6B and Table 2). Since the alanine has shorter sidechain than that of cysteine, and the substitution of channel turret Gln592 by cysteine was found to have intermediate-impact on hERG channel function shown by the differential effects of mutant channel function in the presence or absence of reducing agent
DTT [22]. In combination with the less effect of mutant hERG-Q592A on toxin BmKKx2 affinity (Figures 5G, 6B and Table 2), channel turret Gln592 did not play a critical role in scorpion toxin BeKm-1 or BmKKx2 binding since toxin $\mathrm{BeKm}-1$ or $\mathrm{BmKKx} 2$ had the similar structures and common functional residues (Figure 7A and B).

Further comparison of the pharmacological data of toxin BmKKx2 and BeKm-1 towards mutant hERG channels showed a common feature that three turret regions including $\mathrm{S} 5 \mathrm{H}$, helix and HP played an unimportant role in toxin binding [10] (Figures 4, 5, and 6 and Table 2). In our previous work, the hERG channel turrets were predicted to possibly affect toxin BeKm-1 binding process because of its significant flexibility although they were likely far from the bound toxin BeKm-1 during the molecular dynamic simulations [10]. This inference was supported by about 10-fold drops of toxin BmKKx2 affinities towards mutant hERGR582A, hERG-I583A, hERG-H587A, hERG-Q592A, hERGY597A, hERG-K608A (Figures 5, 6 and Table 2).

In summary, the comprehensive analysis of the pharmacological data of toxin BmKKx2 and BeKm-1 towards mutant hERG channels showed that channel turrets formed an 
Table 2 The binding affinities of toxin BmKKx2 towards wild-type and mutant hERG channel

\begin{tabular}{|c|c|c|c|}
\hline hERG mutant & $\mathrm{IC} 50^{a}$ & $\mathrm{n}^{b}$ & $I C_{50 \text { (mut) }} / I C_{50(w t)}$ \\
\hline & $\mathrm{nM}$ & & \\
\hline Wild Type & $6.7 \pm 1.7$ & 7 & 1.0 \\
\hline H578A & N.E & 5 & $-e$ \\
\hline D580A & L.E $^{d}$ & 5 & - \\
\hline $\mathrm{R} 582 \mathrm{~A}$ & $76.5 \pm 32.6$ & 4 & 11.4 \\
\hline I583A & $87.5 \pm 16.8$ & 4 & 13.1 \\
\hline W585A & N.E & 6 & - \\
\hline L586A & $24.3 \pm 8.4$ & 4 & 3.6 \\
\hline H587A & $59.7 \pm 31.7$ & 5 & 8.9 \\
\hline N588A & $14.5 \pm 4.3$ & 4 & 2.2 \\
\hline L589A & L.E & 6 & - \\
\hline D591A & $19.6 \pm 1.7$ & 4 & 2.9 \\
\hline Q592A & $89.5 \pm 39.8$ & 5 & 13.4 \\
\hline K595A & $65.2 \pm 19.1$ & 5 & 9.7 \\
\hline P596A & $60.8 \pm 23.4$ & 4 & 9.1 \\
\hline Y597A & $118.2 \pm 26.7$ & 4 & 17.6 \\
\hline N598A & $15.2 \pm 4.8$ & 4 & 2.3 \\
\hline S600A & $12.1 \pm 3.5$ & 4 & 1.8 \\
\hline K608A & $116.1 \pm 29.2$ & 5 & 17.3 \\
\hline D609A & L.E. & 8 & - \\
\hline K610A & $6.7 \pm 5.1$ & 5 & 1.0 \\
\hline T613A & $42.6 \pm 17.1$ & 5 & 6.4 \\
\hline N629A & L.E & 7 & - \\
\hline S631A & $698.0 \pm 64.4$ & 5 & 104.2 \\
\hline
\end{tabular}

${ }^{a} \mathrm{IC}_{50}$ value(half-maximum inhibition concentration) represents as Mean \pm Standard Error. ${ }^{b}$ number of independent experiments.

'no expression, can't detect fluorescent flag in all transfected HEK 293 T cells. ${ }^{d}$ low expression, with detectable fluorescent flag but can only elicit weak current in all transfected HEK 293 cells.

$e_{-}$means the lack of $\mathrm{IC}_{50}$ value of mutants.

open conformation in scorpion toxin binding [10] (Figures 5, 6 and Table 2). This open conformation was further shown in the toxin BmKKx2-hERG channel complex through the structural modeling using our previous toxin BeKm-1hERG channel complex as the template [10] (Figure 7C). Such conformational feature of hERG channel turrets was also observed in the turrets of Kv1.2 channel bound by scorpion toxin maurotoxin [6] and the turrets of BKCa channel bound by scorpion toxin ChTX [8].

\section{Conserved function of $h E R G$ channel pore region in toxin BmKKx2 recognition}

The pore regions of potassium channels play an important role during its interactions with the channel-blocking animal toxins $[19,20]$. In this work, the channel Ser631 was found essential for toxin BmKKx2 binding shown by about 104-fold drop of BmKKx2 affinity compared with that of wild-type hERG channel (Figures 5P, 6D and Table 2). As shown in Figure 4, the corresponding residues in the position of Ser631 near hERG channel selectivity filter were critical for toxin activities among different potassium channels [6,19,23], which also rationalized the conserved and critical function of Ser631 in the hERG channel pore region. In the previous cysteine-scanning experiments [11], the importance of Ser631 residue in hERG channel was less significant likely since its substitution by cysteine was found to have intermediate-impact on hERG channel function shown by the differential effects of mutant channel function in the presence or absence of reducing agent DTT [22]. As shown in Figure 7D, the polar and nonpolar interactions between channel Ser631 and toxin residues further illustrated the essential function of channel Ser631 for toxin binding in the modeled hERG channel-toxin BmKKx2 complex structure.

\section{Conclusions}

In this work, we revisited the hERG potassium channel which has an unusual longer turret with the considerable conformational flexibility. With the help of a new specific scorpion toxin BmKKx2, the first specific blocker for hERG channels from scorpion Mesobuthus martensii, the turret role of hERG channel was re-investigated through the alanine-scanning mutagenesis. All pharmacological data indicated that different residue substitutions by alanine decreased the affinities of toxin BmKKx2 by about 10-fold compared with that of wild-type hERG channel, which revealed that channel turret played a secondary role in toxin binding. The further comprehensive analysis of the pharmacological data of scorpion toxins towards mutant hERG channels from the previous cysteine-scanning and present alanine-scanning mutagenesis showed that channel turrets formed an open conformation in scorpion toxin binding. Together, these findings were helpful to understand the unique role of hERG channel turrets in toxin recognition and enrich the diversity of turret structure-function relationships among the different potassium channels.

\section{Methods}

Clone, expression and purification of BmKKx2 peptide

The cDNA library of Mesobuthus martensii was constructed by our group as described previously [21]. cDNAs were cloned into pSPORT1 plasmids and transformed into E. coli $\mathrm{DH} 5 \alpha$ cells (obtained from China Center for Type Culture Collection, CCTCC). Randomly chosen cDNA clones were sequenced to obtain a reliable representation of the toxin content in the venom gland.

After acquiring the full-length cDNA of BmKKx2, expression plasmid pGEX-6p-1-BmKKx2 was constructed using overlap PCR method. The primers used were: forward primer 1: 5'-CCTACAGATATTAAATGCAGTGCA 

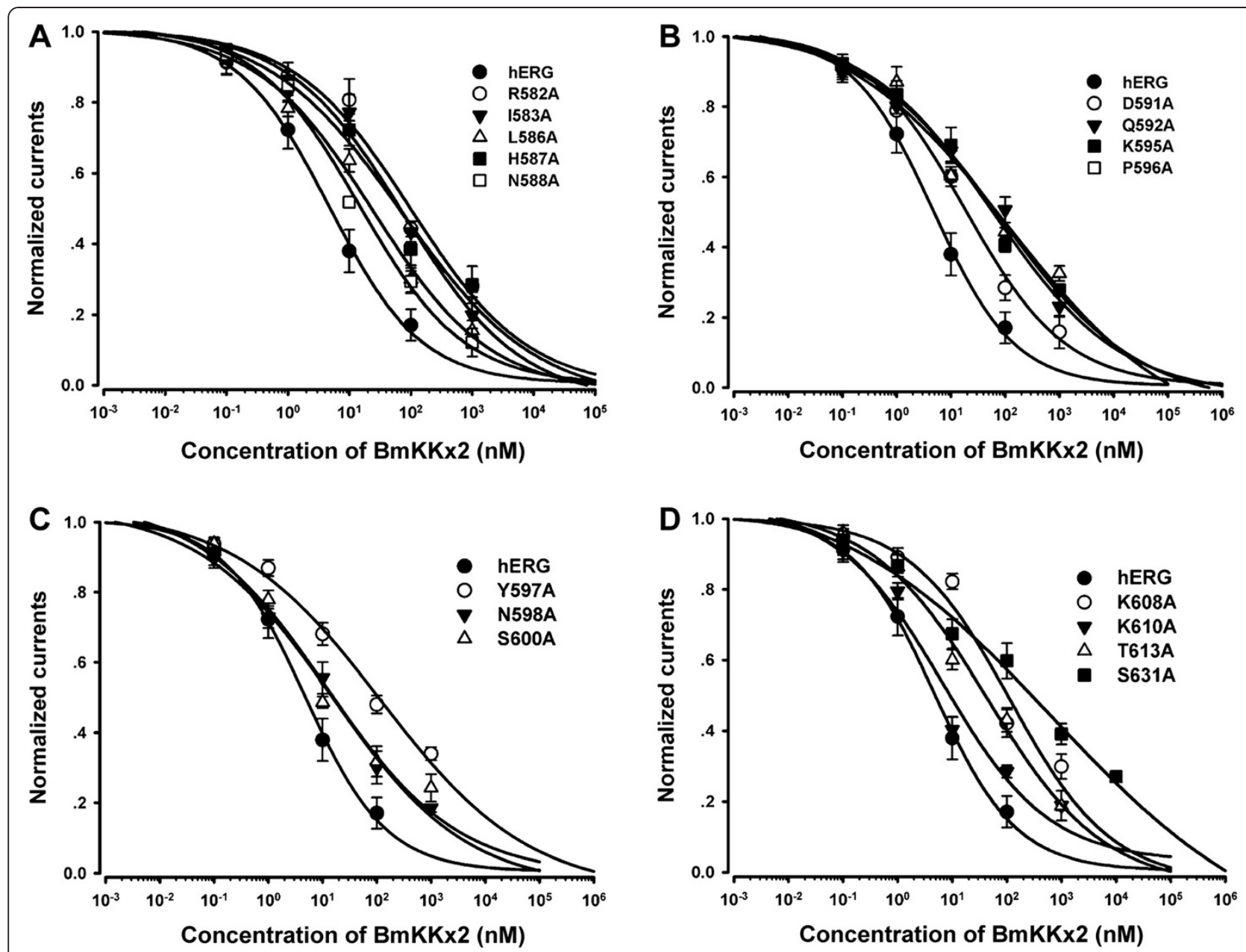

Figure 6 Dose-dependent inhibition of hERG channel mutants by BmKKx2. (A) hERG-R582A, hERG-1583A, hERG-L586A, hERG-H587A and hERG-N588A. (B) hERG-D591A, hERG-Q592A, hERG-K595A and hERG-P596A. (C) hERG-Y597A, hERG-N598A and hERG-S600A. (D) hERG-K608A, hERG-K610A, hERG-T613A and hERG-S631A. The IC 50 values are listed in Table 2.

TCTTATCAATGT- TTCCCTGTTTG-3'; reverse primer 1: 5'-AACGTCCATTAGTCTTTCCGAAGCG-GCTTTT ACAAACAGGGAAACATTG-3'; forward primer 2: 5’GTGAATTCGAT-GACGATGACAAGCGTCCTACAGA TATTAAATG-3'; reverse primer 2: 5'-TAGCTCGAGC TAGAAACAGTCGCATAAACCATTCACGCAACGTCC ATTAG-3'. The restriction enzyme sites are underlined, and an enterokinase cleavage site is in bold. The PCR products were inserted into expression vector pGEX$6 \mathrm{p}-1$ and sequenced with universal pGEX primers. $E$. coli Rosetta (DE3) cells were used to express BmKKx2 and its mutants according to previous techniques of our group $[7,10,15,21]$.

\section{Alanine-scanning mutagenesis of hERG channel}

The wild-type hERG-pEGFP-N2 vector was used in this work. Twenty-two mutants were chosen for study base on the structure analysis of hERG channel and our previous model [10]. All the channel mutants were constructed with the QuickChange site-directed mutagenesis kit (Stratagene), as described by the manufacture. The mutated expression plasmids were confirmed by sequencing after construction (Takara).

\section{Cell culture and transfection}

Human embryonic kidney (HEK293) cells were cultured in Dulbecco's Modified Eagle's Medium (DMEM, Gibco) with $10 \%$ Fetal Calf Serum (Invitrogen Life Technologies, Carlesbad, CA), supplemented with 100 units $/ \mathrm{mL}$ ampicillin and $100 \mu \mathrm{g} / \mathrm{mL}$ streptomycin in a humidified $5 \% \mathrm{CO}_{2}$ incubator at $37^{\circ} \mathrm{C}$.

Cells were transfected with $6 \mu \mathrm{g}$ plasmid of wild-type or mutant hERG using the FuGene Transfection Method (Roche Diagnostics, Switzerland). Currents were recorded 24-48 hours after transfection in EGFP fluorescent cells at room temperature using the whole-cell patch-clamp mode with an EPC10 Amplifier (HEKA, Germany). 

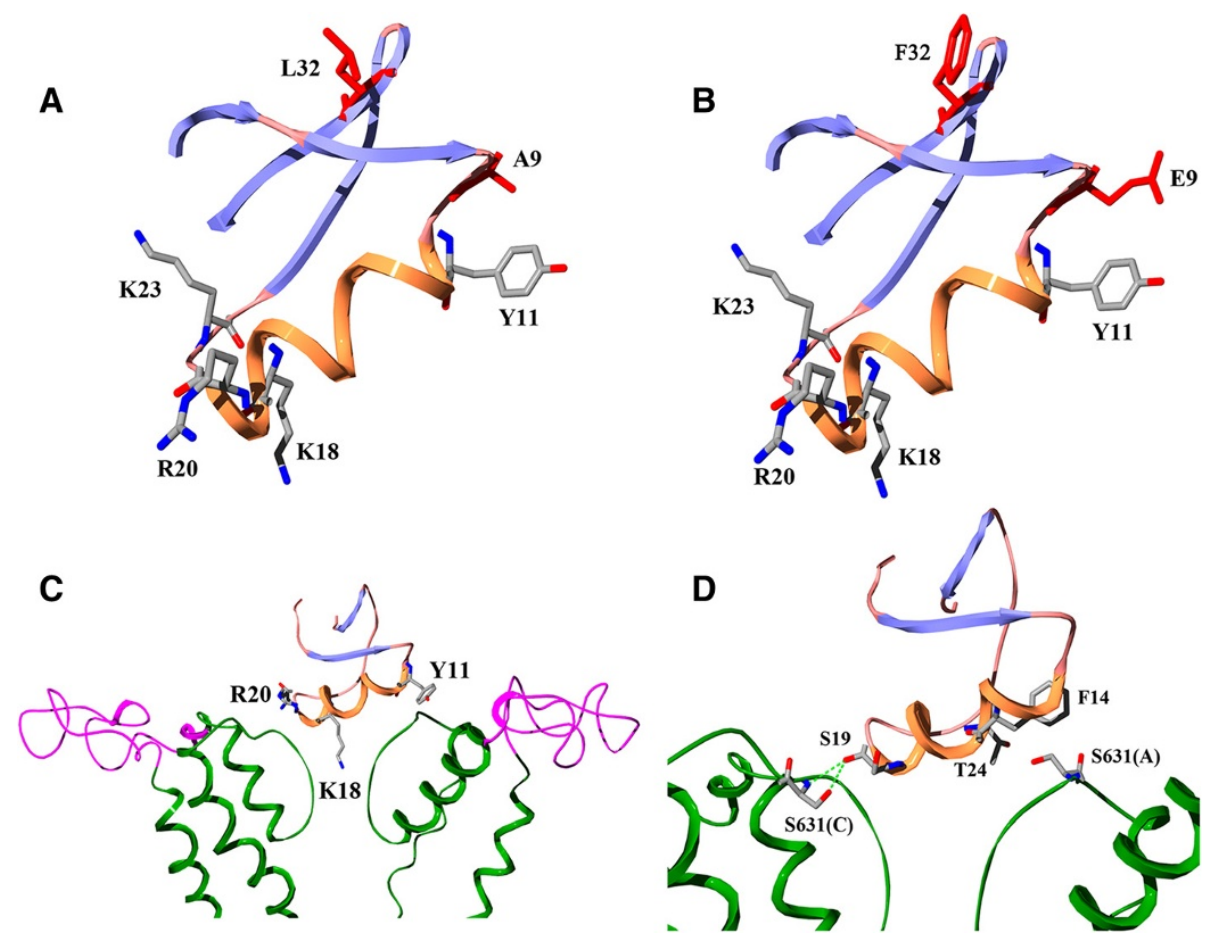

Figure 7 Stuctural model and analysis of toxin BmKKx2 and its complex with hERG channel. (A-B) The distribution of functional residues and differential residues between BmKKx2 and BeKm-1. (A) BmKKx2, (B) BeKm-1. (C) The spatial orientation of hERG channel turrets and toxin BmKKx2 in their complex structural. (D) Interaction details between hERG channel Ser 631 residues and toxin residues.

\section{Electrophysiology and data analysis}

Cells expressing $\mathrm{Kv}$ channels were incubated in the external solution consisted of (in $\mathrm{mM}$ ): $137 \mathrm{NaCl}, 4 \mathrm{KCl}, 1$ $\mathrm{MgCl}_{2}, 1.8 \mathrm{CaCl}_{2}, 10 \mathrm{D}$-Glucose and 10 HEPES (pH7.4 with $\mathrm{NaOH}$ ); and the internal solution contained (in $\mathrm{mM}): 130 \mathrm{KCl}, 1 \mathrm{MgCl}_{2}, 5 \mathrm{MgATP}, 5$ EGTA, 10 HEPES (pH 7.2 with $\mathrm{KOH})$. For Kv1 channels and Kv4.2, currents were elicited by $200-\mathrm{ms}$ or $500-\mathrm{ms}$ depolarizing pulses from a holding potential of -80 to $50 \mathrm{mV}$, individually. While cells expressing hERG channel were depolarized from a holding potential of $-80 \mathrm{mV}$ to $+40 \mathrm{mV}$ for $500 \mathrm{~ms}$ then hyperpolarized to $-120 \mathrm{mV}$ for $1 \mathrm{~s}$ and current amplitudes were measured from the peak inward current at $-120 \mathrm{mV}$.

For the recording of SKCa channels, the bath solution contained $130 \mathrm{mM}$ sodium aspartate, $30 \mathrm{mM} \mathrm{K}^{+}$-aspartate, $2 \mathrm{mM} \mathrm{CaCl}_{2}, 1 \mathrm{mM} \mathrm{MgCl}$, and $10 \mathrm{mM}$ HEPES (pH 7.4 with $\mathrm{NaOH}$ ). The pipette solution contained $145 \mathrm{mM} \mathrm{K}^{+}$-aspartate, $8.7 \mathrm{mM} \mathrm{CaCl} 2,2 \mathrm{mM} \mathrm{MgCl}_{2}$, $10 \mathrm{mM}$ EGTA, and $10 \mathrm{mM}$ HEPES (pH 7.2 with $\mathrm{KOH}$ ) to achieve an intracellular free $\mathrm{Ca}^{2+}$ concentration of $1 \mu \mathrm{M}$. The membrane potentials were clamped to $-120 \mathrm{mV}$ for $50 \mathrm{~ms}$ (which was used for the current measurements), followed by a $400 \mathrm{~ms}$ voltage ramp from -120 to $+60 \mathrm{mV}$ and were kept for $5 \mathrm{~s}$ between ramps at $-40 \mathrm{mV}$.

When toxin peptides were applied, $0.01 \%$ Bovine Serum Albumin (BSA) was added to the external solution to seal the tubes of the perfusion system. A multi-channel micro-perfusion system MPS-2 (INBIO Inc, Wuhan, China) was used to exchange the external recording bath solution. Current amplitudes were obtained using Pulse as acquisition software while data was analyzed with Sigmaplot 11.0 (SPSS Inc. USA). Dose response curves for the toxin concentration dependence of current inhibition were fitted with a modified Hill equation,

$$
\mathrm{I}_{\text {toxin }} / \mathrm{I}_{\text {control }}=1 /\left(1+[\mathrm{T}] / \mathrm{IC}_{50}\right),
$$

where $\mathrm{IC}_{50}$ is the half-maximum inhibition concentration, I is the peak tail current, $[\mathrm{T}]$ represents the concentration of toxins. Results are shown as mean \pm S.E., and $n$ is the number of experiments.

\section{Additional file}

Additional file 1: Figure S1. Circular dichroism spectra of wild-type BmKK $\times 2$ and mutants. (A) Circular dichroism spectra of recombinant BmKKx2, BmKKx2-R1A, BmKKx2-Y11A, BmKKx2-K23A and BmKKx2-F32A. (B) Circular dichroism spectra of BmKKx2, BmKKx2-K18A and BmKKx2-R20A. The measurement was carried out in the UV range of $250-190 \mathrm{~nm}$ at $25^{\circ} \mathrm{C}$ in water on a Jasco-810 spectropolarimeter with a concentration of $0.2-0.4 \mathrm{mg} / \mathrm{mL}$.

\section{Competing interests}

The authors declare that they have no competing interests. 


\section{Authors' contributions}

YTH, WXL and YLW designed the study. YTH and TL constructed the mutant channels. JJW was responsible for harvesting recombinated BmKK 2 2 peptide and its mutants. YTH carried out cell culture and transfection. YTH and JF performed electrophysiological experiments. YTH and JH analyzed data. YTH and YLW wrote the manuscript. All authors read and approved the final manuscript.

\section{Acknowledgements}

This work was supported by grants from the National Basic Research Program of China (No. 2010CB529800), National High Technology Research and Development Program of China (No. 2012AA020304), the National Natural Sciences Foundation of China (No.31170789), the New Century Excellent Talents in Wuhan University by the Ministry of Education of China (No. NCET-10-0651) and Wuhan City Science and Technology Foundation of China (No. 2013070204020046)

\section{Author details}

'State Key Laboratory of Virology, College of Life Sciences, Wuhan University, Wuhan 430072, China. ${ }^{2}$ Research Center of Ion Channelopathy, Institute of Cardiovascular Diseases, Union Hospital, Tongji Medical College, Huazhong University of Science and Technology, Wuhan 430022, China.

Received: 3 January 2014 Accepted: 11 February 2014

Published: 11 April 2014

\section{References}

1. Ashcroft FM: From molecule to malady. Nature 2006, 440:440-447.

2. Shieh CC, Coghlan M, Sullivan JP, Gopalakrishnan M: Potassium channels: molecular defects, diseases, and therapeutic opportunities. Pharmacol Rev 2000, 52:557-594.

3. Long SB, Campbell EB, Mackinnon R: Crystal structure of a mammalian voltage-dependent Shaker family $\mathrm{K}^{+}$channel. Science 2005, 309:897-903.

4. Tao X, Avalos JL, Chen J, MacKinnon R: Crystal structure of the eukaryotic strong inward-rectifier $\mathrm{K}^{+}$channel Kir2.2 at $3.1 \AA$ \& resolution. Science 2009, 326:1668-1674.

5. Whorton MR, Mackinnon R: X-ray structure of the mammalian GIRK2betagamma G-protein complex. Nature 2013, 498:190-197.

6. Yi H, Qiu S, Cao ZJ, Wu YL, Li WX: Molecular basis of inhibitory peptide maurotoxin recognizing Kv1.2 channel explored by ZDOCK and molecular dynamic simulations. Proteins 2008, 70:844-854.

7. Feng J, Hu YT, Yi H, Yin SJ, Han S, Hu J, Chen ZY, Yang WS, Cao ZJ, De Waard M, Sabatier JM, Li WX, Wu YL: Two conserved arginine residues from the SK3 potassium channel outer vestibule control selectivity of recognition by scorpion toxins. J Biol Chem 2013, 288:12544-12553.

8. Gan GL, Yi H, Chen MR, Sun L, Li WX, Wu YL, Ding JP: Structural basis for toxin resistance of beta4-associated calcium-activated potassium (BK) channels. J Biol Chem 2008, 283:24177-24184.

9. Torres AM, Bansal PS, Sunde M, Clarke CE, Bursill JA, Smith DJ, Bauskin A Breit SN, Campbell TJ, Alewood PF, Kuchel PW, Vandenberg Jl: Structure of the HERG K ${ }^{+}$channel S5P extracellular linker: role of an amphipathic alpha-helix in C-type inactivation. J Biol Chem 2003, 278:42136-42148.

10. Yi H, Cao ZJ, Yin SJ, Dai C, Wu YL, Li WX: Interaction simulation of hERG $\mathrm{K}^{+}$ channel with its specific BeKm-1 peptide: insights into the selectivity of molecular recognition. J Proteome Res 2007, 6:611-620.

11. Tseng GN, Sonawane KD, Korolkova W, Zhang M, Liu J, Grishin EV, Guy HR: Probing the outer mouth structure of the HERG channel with peptide toxin footprinting and molecular modeling. Biophys $J$ 2007, 92:3524-3540.

12. Korolkova YV, Kozlov SA, Lipkin AV, Pluzhnikov KA, Hadley JK, Filippov AK, Brown DA, Angelo K, Strobaek D, Jespersen T, Olesen SP, Jensen BS, Grishin EV: An ERG channel inhibitor from the scorpion Buthus eupeus. J Biol Chem 2001, 276:9868-9876.

13. Zeng XC, Luo F, Li WX: Molecular dissection of venom from Chinese scorpion Mesobuthus martensii: identification and characterization of four novel disulfide-bridged venom peptides. Peptides 2006, 27:1745-1754.

14. Peter M Jr, Varga Z, Hajdu P, Gaspar R Jr, Damjanovich S, Horjales E, Possani $L D$, Panyi G: Effects of toxins Pi2 and Pi3 on human T lymphocyte Kv1.3 channels: the role of Glu7 and Lys24. J Membr Biol 2001, 179:13-25.

15. Han S, Yin SJ, Yi H, Mouhat S, Qiu S, Cao ZJ, Sabatier JM, Wu YL, Li WX: Protein-protein recognition control by modulating electrostatic interactions. J Proteome Res 2010, 9:3118-3125.
16. Peigneur S, Beress L, Moller C, Mari F, Forssmann WG, Tytgat J: A natural point mutation changes both target selectivity and mechanism of action of sea anemone toxins. FASEB J 2012, 26:5141-5151.

17. Korolkova YV, Bocharov EV, Angelo K, Maslennikov IV, Grinenko OV, Lipkin AV, Nosyreva ED, Pluzhnikov KA, Olesen SP, Arseniev AS, Grishin EV: New binding site on common molecular scaffold provides hERG channel specificity of scorpion toxin BeKm-1. J Biol Chem 2002, 277:43104-43109.

18. Jiang M, Zhang M, Maslennikov IV, Liu J, Wu DM, Korolkova YV, Arseniev AS, Grishin EV, Tseng GN: Dynamic conformational changes of extracellular S5-P linkers in the hERG channel. J Physiol 2005, 569:75-89.

19. Gilquin B, Braud S, Eriksson MA, Roux B, Bailey TD, Priest BT, Garcia ML, Menez A, Gasparini S: A variable residue in the pore of Kv1 channels is critical for the high affinity of blockers from sea anemones and scorpions. J Biol Chem 2005, 280:27093-27102.

20. Yin SJ, Jiang L, Yi H, Han S, Yang DW, Liu ML, Liu H, Cao ZJ, Wu YL, Li WX: Different residues in channel turret determining the selectivity of ADWX-1 inhibitor peptide between Kv1.1 and Kv1.3 channels. J Proteome Res 2008, 7:4890-48977.

21. Han S, Yi H, Yin SJ, Chen ZY, Liu H, Cao ZJ, Wu YL, Li WX: Structural basis of a potent peptide inhibitor designed for Kv1.3 channel, a therapeutic target of autoimmune disease. J Biol Chem 2008, 283:19058-19065.

22. Liu J, Zhang M, Jiang M, Tseng GN: Structural and functional role of the extracellular s5-p linker in the HERG potassium channel. J Gen Physiol 2002, 120:723-737.

23. Visan V, Fajloun Z, Sabatier JM, Grissmer S: Mapping of maurotoxin binding sites on hKv1.2, hKv1.3, and hlKCa1 channels. Mol Pharmacol 2004, 66:1103-1112.

doi:10.1186/2045-3701-4-18

Cite this article as: Hu et al:: Open conformation of $h E R G$ channel turrets revealed by a specific scorpion toxin BmKKx2. Cell \& Bioscience 2014 4:18.

\section{Submit your next manuscript to BioMed Central and take full advantage of:}

- Convenient online submission

- Thorough peer review

- No space constraints or color figure charges

- Immediate publication on acceptance

- Inclusion in PubMed, CAS, Scopus and Google Scholar

- Research which is freely available for redistribution 